Dedicated to the bright memory of

Alexey Borisovich Shabat, who is one of the main creators of modern theory of classical integrable systems

doi:10.13108/2021-13-2-81

\title{
FINITE-GAP SOLUTIONS OF NONLOCAL EQUATIONS IN ABLOWITZ-KAUP-NEWELL-SEGUR HIERARCHY
}

\author{
A.O. SMIRNOV, V.B. MATVEEV
}

\begin{abstract}
Nonlinear nonlocal models exist in many fields of physics. The most known of them are models possessing $\mathcal{P} \mathcal{T}$-symmetries. Apart of $\mathcal{P} \mathcal{T}$-symmetric models, nonlocal models with inverse time and/or coordinates are actively studied. Other types of nonlocalities arise much rare. As a rule, in works devoted to nonlinear nonlocal equations, soliton or quasi-rational solutions to such equations are studied.

In the present work we consider nonlocal symmetries, to which all equations in the Ablowitz-Kaup-Newell-Segur hierarchy. On the base of the properties of solutions satisfying nonlocal reductions of the equations in the Ablowitz-Kaup-Newell-Segur hierarchy, we propose a modification of theta-functional formula for Baker-Akhiezer functions. We find the conditions for the parameters of spectral curves associated with multi-phase solutions possessing no exponential growth at infinity. We show that under these conditions, the variables separate. The most part of statement of our work remain true for soliton and quasi-rational solutions since they are limiting cases for the multi-phase solutions.
\end{abstract}

Keywords: Nonlinear Schrödinger equation, Ablowitz-Kaup-Newell-Segur hierarchy, nonlocal equation, PT-symmetry, finite-gap solution, spectral curve, theta function.

Mathematics Subject Classification: 37K10, 35Q55, 35Q60

\section{INTRODUCTION}

Nonlinear nonlocal models arise in many areas of physics. The most famous of them are models with $\mathcal{P} \mathcal{T}$-symmetry. For a general understanding of the role of $\mathcal{P} \mathcal{T}$-symmetry in a wide range of physical problems related to the spectral theory of non-Hermitian operators with real spectra, its manifestations in the theory of nonlinear waves in various physical media and, in particular, in the theory of nonlocal integrable systems, we can recommend survey [1] and recent book [2].

After the publication of works by Ablowitz and Musslimani [3]- [8], the attention to solutions of nonlocal integrable nonlinear equations sharply increased, see, for example, $|9|-|30|$. As a rule, in these works, the authors used the Darboux transformation or Hirota method for constructing solutions. Then there arose a natural question on the possibility of constructing solutions of nonlocal integrable equations by the method of finite-zone integration. Our first results on the theory of finite-zone solutions of nonlocal integrable equations from the AKNS

A.O. Smirnov, V.B. Matveev, Finite-gap solutions of nonlocal equations in Ablowitz-KaupNewell-Segur hierarchy.

(C) Smirnov A.O., Matveev V.B. 2021.

The research is supported by RFBR (grant no. 19-01-00734) and the Ministry of Science and Higher Education of Russian Federation (agreement no. FSRF-2020-0004).

Submitted March 15, 2021. 
hierarchy were published in papers $[31]-[33 \mid$. In this paper, we summarize our research on this topic.

The present work consists of five sections. In the first section, following [34], [35], we derive equations of the AKNS hierarchy and analyze their symmetries. The second section is devoted to our proposed modification of the Baker-Akhiezer function. As a base, the Baker-Akhiezer function for the classical versions of the nonlinear Schrödinger equation serves [36]- [38]. In the end of the second section, we provide formulae for finite-zone solutions corresponding to the proposed Baker-Akhiezer function. In Section 3, we study the properties of finite-gap solutions constructed by three classes of spectral curves with anti-holomorphic involution. In the general case, finite-zone solutions constructed by spectral curves with anti-holomoric involution have exponentially grow/decay as the independent arguments tend to positive/negative infinity. Because of this, we impose, an additional condition on the spectral curves assuming the presence of a holomorphic involution. Sections 4 and 5 show how the presence of this holomorphic involution affects the parameters of the finite-zone solutions of the nonlocal AKNS hierarchy equations constructed in Section 2. In particular, in Section 5, we show that the presence of a holomorphic involution leads to the separation of variables: each theta function of a finite-zone solution is a sum of products of two theta functions of lower dimension. The independent variables of one of the smaller theta functions contain times with an odd index $t_{1}, t_{3}, \ldots$, while the other theta function depends on the variable $x$ and times with an even index $t_{2}, t_{4}, \ldots$ Section 5 also provides examples of two-zone solutions of nonlocal AKNS hierarchy equations expressed in terms of one-dimensional theta functions.

\section{EQUATIONS IN AKNS HIERARCHY}

It is well known that equations in the AKNS hierarchy 39 are obtained as a result of joint consideration of the equations

where, see, for instance, |34], |35|,

$$
\left\{\begin{array}{l}
\Psi_{x}=\mathfrak{U} \Psi \\
\Psi_{t_{k}}=\mathfrak{V}_{k} \Psi
\end{array}\right.
$$

$$
\begin{gathered}
\mathfrak{U}:=\lambda J+\mathfrak{U}^{0}, \quad \mathfrak{V}_{1}:=2 \lambda \mathfrak{U}+\mathfrak{V}_{1}^{0}, \quad \mathfrak{V}_{k+1}:=2 \lambda \mathfrak{V}_{k}+\mathfrak{V}_{k+1}^{0}, \quad k \geqslant 1, \\
J:=\left(\begin{array}{cc}
-i & 0 \\
0 & i
\end{array}\right), \quad \mathfrak{U}^{0}:=\left(\begin{array}{cc}
0 & i p \\
-i q & 0
\end{array}\right), \\
\mathfrak{V}_{k}^{0}=\left(\begin{array}{cc}
-i^{k} F_{k}(p, q) & i^{k-1} H_{k}(p, q) \\
i^{k-1} G_{k}(p, q) & i^{k} F_{k}(p, q)
\end{array}\right) .
\end{gathered}
$$

The equation

$$
\left(\Psi_{x}\right)_{t_{k}}=\left(\Psi_{t_{k}}\right)_{x}
$$

implies the following recurrent relations for the functions $F_{k}(p, q), H_{k}(p, q)$ and $G_{k}(p, q)$ :

$$
\begin{aligned}
& H_{1}(p, q)=-p_{x}, \quad G_{1}(p, q)=-q_{x}, \\
& \left(F_{k}(p, q)\right)_{x}=-p G_{k}(p, q)-q H_{k}(p, q), \\
& H_{k+1}(p, q)=2 p F_{k}(p, q)+\left(H_{k}(p, q)\right)_{x}, \\
& G_{k+1}(p, q)=-2 q F_{k}(p, q)-\left(G_{k}(p, q)\right)_{x} .
\end{aligned}
$$

In particular,

$$
\begin{aligned}
& F_{1}(p, q)=p q, \quad H_{2}(p, q)=2 p^{2} q-p_{x x} \\
& G_{2}(p, q)=-2 q^{2} p+q_{x x}, \quad F_{2}(p, q)=p_{x} q-p q_{x} \\
& H_{3}(p, q)=6 p q p_{x}-p_{x x x}, \quad G_{3}(p, q)=6 p q q_{x}-q_{x x x}
\end{aligned}
$$




$$
\begin{aligned}
F_{3}(p, q)= & p q_{x x}+q p_{x x}-p_{x} q_{x}-3 p^{2} q^{2} \\
H_{4}(p, q)= & -6 p^{3} q^{2}+6 q p_{x}^{2}+4 p p_{x} q_{x}+8 p q p_{x x}+2 p^{2} q_{x x}-p_{x x x x}, \\
G_{4}(p, q)= & 6 p^{2} q^{3}-6 p q_{x}^{2}-4 q p_{x} q_{x}-8 p q q_{x x}-2 q^{2} p_{x x}+q_{x x x x} \\
F_{4}(p, q)= & -6 p q^{2} p_{x}+6 p^{2} q q_{x}-q_{x} p_{x x}+p_{x} q_{x x}+q p_{x x x}-p q_{x x x}, \\
H_{5}(p, q)= & -30 p^{2} q^{2} p_{x}+10 p_{x}^{2} q_{x}+20 q p_{x} p_{x x}+10 p q_{x} p_{x x} \\
& +10 p p_{x} q_{x x}+10 p q p_{x x x}-p_{x x x x x} \\
G_{5}(p, q)= & -30 p^{2} q^{2} q_{x}+10 p_{x} q_{x}^{2}+10 q q_{x} p_{x x}+10 q p_{x} q_{x x} \\
& +20 p q_{x} q_{x x}+10 p q q_{x x x}-q_{x x x x x}, \\
F_{5}(p, q)= & 10 p^{3} q^{3}-5 q^{2} p_{x}^{2}-5 p^{2} q_{x}^{2}-10 p q^{2} p_{x x}-10 p^{2} q q_{x x}+p_{x x} q_{x x} \\
& -q_{x} p_{x x x}-p_{x} q_{x x x}+q p_{x x x x}+p q_{x x x x} .
\end{aligned}
$$

It is easy to show that the functions $F_{k}(p, q), H_{k}(p, q)$ and $G_{k}(p, q)$ possess the following properties [34, |35]

$$
\begin{gathered}
F_{k}(q, p)=(-1)^{k-1} F_{k}(p, q), \quad F_{k}(-p,-q)=F_{k}(p, q), \\
G_{k+1}(p, q)=(-1)^{k} H_{k+1}(q, p), \quad H_{k+1}(-p,-q)=-H_{k+1}(p, q)
\end{gathered}
$$

and

$$
\begin{aligned}
& F_{k}\left(\left.p\right|_{x=-x},\left.q\right|_{x=-x}\right)=\left.(-1)^{k-1} F_{k}(p, q)\right|_{x=-x}, \\
& G_{k}\left(\left.p\right|_{x=-x},\left.q\right|_{x=-x}\right)=\left.(-1)^{k} G_{k}(p, q)\right|_{x=-x}, \\
& H_{k}\left(\left.p\right|_{x=-x},\left.q\right|_{x=-x}\right)=\left.(-1)^{k} H_{k}(p, q)\right|_{x=-x} .
\end{aligned}
$$

An implication of the compatibility conditions are also integrable nonlinear evolutionary AKNS hierarchy equations being of the form

$$
p_{t_{k}}=-i^{k} H_{k+1}(p, q), \quad q_{t_{k}}=-i^{k} G_{k+1}(p, q)
$$

or

$$
p_{t_{k}}+i^{k} H_{k+1}(p, q)=0, \quad q_{t_{k}}+(-i)^{k} H_{k+1}(q, p)=0 .
$$

In our notations, classical integrable nonlinear equations have the following form:

1. focusing nonlinear Schrödinger equation

$$
i p_{t_{1}}-H_{2}\left(p,-p^{*}\right)=0
$$

2. defocusing nonlinear Schrödinger equation

$$
i p_{t_{1}}-H_{2}\left(p, p^{*}\right)=0 ;
$$

3. real modified Korteweg-de Vries equation

$$
p_{t_{2}}-H_{3}(p, \pm p)=0
$$

4. Lakshmanan-Porsezian-Daniel equation ( $\left.40-42, t=-t_{3}\right)$

$$
i p_{t}-H_{4}\left(p,-p^{*}\right)=0 \text {. }
$$

\section{BAKER-AKHIEZER FUNCTION FOR NONLOCAL EQUATIONS}

We change the spectral parameter $\lambda \rightarrow i \lambda$ in equations 1.2 :

$$
\mathfrak{U}:=i \lambda J+\mathfrak{U}^{0}, \quad \mathfrak{V}_{1}:=2 i \lambda \mathfrak{U}+\mathfrak{V}_{1}^{0}, \quad \mathfrak{V}_{k+1}:=2 i \lambda \mathfrak{V}_{k}+\mathfrak{V}_{k+1}^{0}, \quad k \geqslant 1 .
$$

It is easy to understand that the compatibility conditions for the Lax pairs (1.1) remain the same in this case, although the reality conditions changes, as well as the reductions containing the complex conjugation. 
Following [36], [37], see also [34], |35], [43]- [45], we define a hyperelliptic curve $\Gamma=\{(\chi, \lambda)\}$ of genus $g$

$$
\Gamma: \quad \chi^{2}=\prod_{j=1}^{2 g+2}\left(\lambda-\lambda_{j}\right) \equiv \lambda^{2 g+2}+\sum_{j=1}^{2 g+2} \chi_{j} \lambda^{2 g+2-j}, \quad \chi_{j} \in \mathbb{R} .
$$

On $\Gamma$, we choose a canonical basis of cycles $\gamma^{t}=\left(a_{1}, \ldots, a_{g}, b_{1}, \ldots, b_{g}\right)$ with matrix of intersection indices

$$
C_{0}=\left(\begin{array}{cc}
0 & I \\
-I & 0
\end{array}\right)
$$

On $\Gamma$ we also choose a normalized basis of holomorphic differentials

$$
\begin{gathered}
d \mathcal{U}_{j}=\sum_{k=1}^{g} c_{j k} \lambda^{g-k} \frac{d \lambda}{\chi}, \\
\oint_{a_{k}} d \mathcal{U}_{j}=\delta_{k j}, \quad k, j=1, \ldots, g
\end{gathered}
$$

with the matrix of periods

$$
B_{k j}=\oint_{b_{k}} d \mathcal{U}_{j}, \quad k, j=1, \ldots, g, \quad B^{t}=B, \quad \operatorname{Im}(B)>0 .
$$

By the matrix of periods, we construct a $g$-dimensional theta functions with characteristics $\boldsymbol{\eta}, \boldsymbol{\zeta} \in \mathbb{R}^{g} 46-51$ :

$$
\begin{gathered}
\Theta\left[\boldsymbol{\eta}^{t} ; \boldsymbol{\zeta}^{t}\right](\mathbf{p} \mid B)=\sum_{\mathbf{m} \in \mathbb{Z}^{g}} \exp \left\{\pi i(\mathbf{m}+\boldsymbol{\eta})^{t} B(\mathbf{m}+\boldsymbol{\eta})+2 \pi i(\mathbf{m}+\boldsymbol{\eta})^{t}(\mathbf{p}+\boldsymbol{\zeta})\right\} \\
\Theta\left[\mathbf{0}^{t} ; \mathbf{0}^{t}\right](\mathbf{p} \mid B) \equiv \Theta(\mathbf{p} \mid B) \equiv \Theta(\mathbf{p})
\end{gathered}
$$

where $\mathbf{p} \in \mathbb{C}^{g}$ and the summation is made over the integer $g$-dimensional lattice.

On $\Gamma$ we also define normalized Abelian integrals $\Omega_{j}(\mathcal{P})$ of second kind and Abelian integrals $\omega_{0}(\mathcal{P})$ of third kind with the following asymptotics at infinite points $\mathcal{P}_{\infty}^{ \pm}$:

$$
\begin{array}{ll}
\oint_{a_{k}} d \Omega_{j}=\oint_{a_{k}} d \omega_{0}=0, & k=1, \ldots, g, \\
\Omega_{j}(\mathcal{P})= \pm\left((2 i)^{j-1} \lambda^{j}-K_{j}+O\left(\lambda^{-1}\right)\right), & \mathcal{P} \rightarrow \mathcal{P}_{\infty}^{ \pm}, \\
\omega_{0}(\mathcal{P})=\mp\left(\ln \lambda-\ln K_{0}+O\left(\lambda^{-1}\right)\right), & \mathcal{P} \rightarrow \mathcal{P}_{\infty}^{ \pm}, \\
\chi= \pm\left(\lambda^{g+1}+O\left(\lambda^{g}\right)\right), & \mathcal{P} \rightarrow \mathcal{P}_{\infty}^{ \pm} .
\end{array}
$$

By $2 \pi i \mathbf{V}^{j}$ we denote the vectors of $b$-periods of Abelian integrals $\Omega_{j}(\mathcal{P})$ of second kind.

Following [36], [37] and [38], we define a single-valued vector Baker-Akhiezer function depending on a point $\mathcal{P} \in \Gamma$ :

$$
\Psi(\mathcal{P}, \mathbf{x})=\left(\begin{array}{l}
\psi(\mathcal{P}, \mathbf{x}) \\
\phi(\mathcal{P}, \mathbf{x})
\end{array}\right)
$$

where $\mathbf{x}=\left(x, t_{1}, t_{2}, \ldots\right)^{t}$,

$$
\begin{aligned}
& \psi(\mathcal{P}, \mathbf{x})=r_{1}(\mathbf{x}) \frac{\Theta\left(\mathcal{U}(\mathcal{P})-\mathbf{Z}_{0}+\mathbf{U}(\mathbf{x})\right)}{\Theta\left(\mathcal{U}(\mathcal{P})-\mathbf{Z}_{0}\right)} \exp \{\Omega(\mathcal{P}, \mathbf{x})\} \\
& \left.\phi(\mathcal{P}, \mathbf{x})=r_{2}(\mathbf{x}) \frac{\Theta\left(\mathcal{U}(\mathcal{P})-\mathbf{Z}_{0}+\mathbf{U}(\mathbf{x})+\mathbf{\Delta}\right)}{\Theta\left(\mathcal{U}(\mathcal{P})-\mathbf{Z}_{0}\right)} \exp \left\{\omega_{0}(\mathcal{P})+\Omega(\mathcal{P}, \mathbf{x})\right)\right\}
\end{aligned}
$$


Here $r_{j}$ are normalizing factors, $\boldsymbol{\Delta}$ is the vector of Abelian holomorphic integrals calculated along the paths connecting the points $\mathcal{P}_{\infty}^{-}$and $\mathcal{P}_{\infty}^{+}$and intersecting none of basis cycles,

$$
\begin{aligned}
& \Delta=\mathcal{U}\left(\mathcal{P}_{\infty}^{+}\right)-\mathcal{U}\left(\mathcal{P}_{\infty}^{-}\right), \quad \mathbf{U}(\mathbf{x})=\mathbf{V}^{1} x+\sum_{j \geqslant 1} \mathbf{V}^{j+1} t_{j}, \\
& \Omega(\mathcal{P}, \mathbf{x})=x \Omega_{1}(\mathcal{P})+\sum_{j \geqslant 1} t_{j} \Omega_{j+1}(\mathcal{P}),
\end{aligned}
$$

$\mathbf{Z}_{0} \in \mathbb{C}^{g}$ is the vector defining the initial phase.

We find the normalizing factors

$$
\begin{aligned}
& r_{1}(\mathbf{x})=\rho_{1} \frac{\Theta\left(\mathcal{U}\left(\mathcal{P}^{+}\right)-\mathbf{Z}_{0}\right)}{\Theta\left(\mathcal{U}\left(\mathcal{P}^{+}\right)-\mathbf{Z}_{0}+\mathbf{U}(\mathbf{x})\right)} \exp \left\{K_{1} x+\sum_{j \geqslant 1} K_{j+1} t_{j}\right\}, \\
& r_{2}(\mathbf{x})=K_{0} \rho_{2} \frac{\Theta\left(\mathcal{U}\left(\mathcal{P}^{-}\right)-\mathbf{Z}_{0}\right)}{\Theta\left(\mathcal{U}\left(\mathcal{P}^{-}\right)-\mathbf{Z}_{0}+\mathbf{U}(\mathbf{x})+\Delta\right)} \exp \left\{-K_{1} x-\sum_{j \geqslant 1} K_{j+1} t_{j}\right\},
\end{aligned}
$$

by the asymptotics of vector function $(2.9)$ in the vicinity of infinite points $\mathcal{P}_{\infty}^{ \pm}$:

$$
\begin{array}{ll}
\psi(\mathcal{P}, \mathbf{x})=\left(\rho_{1}+\sum_{j \geqslant 1} \alpha_{j}^{+}(\mathbf{x}) \lambda^{-j}\right) \exp \left\{x \lambda+\sum_{j \geqslant 1} t_{j}(2 i)^{j} \lambda^{j+1}\right\}, & \mathcal{P} \rightarrow \mathcal{P}_{\infty}^{+}, \\
\phi(\mathcal{P}, \mathbf{x})=\lambda^{-1}\left(s_{2}(\mathbf{x})+\sum_{j \geqslant 1} \beta_{j}^{+}(\mathbf{x}) \lambda^{-j}\right) \exp \left\{x \lambda+\sum_{j \geqslant 1} t_{j}(2 i)^{j} \lambda^{j+1}\right\}, & \mathcal{P} \rightarrow \mathcal{P}_{\infty}^{+}, \\
\psi(\mathcal{P}, \mathbf{x})=\left(s_{1}(\mathbf{x})+\sum_{j \geqslant 1} \alpha_{j}^{-}(\mathbf{x}) \lambda^{-j}\right) \exp \left\{-x \lambda-\sum_{j \geqslant 1} t_{j}(2 i)^{j} \lambda^{j+1}\right\}, & \mathcal{P} \rightarrow \mathcal{P}_{\infty}^{-}, \\
\phi(\mathcal{P}, \mathbf{x})=\lambda\left(\rho_{2}+\sum_{j \geqslant 1} \beta_{j}^{-}(\mathbf{x}) \lambda^{-j}\right) \exp \left\{-x \lambda-\sum_{j \geqslant 1} t_{j}(2 i)^{j} \lambda^{j+1}\right\}, & \mathcal{P} \rightarrow \mathcal{P}_{\infty}^{-} .
\end{array}
$$

Theorem 2.1. Algebraic-geometric solutions of equations in AKNS hierarchy constructed by Baker-Akhiezer function (2.9) read as

$$
\begin{aligned}
& p(\mathbf{x})=\frac{2 i A \rho_{1}}{\rho_{2}} \frac{\Theta\left(\mathcal{U}\left(\mathcal{P}_{\infty}^{+}\right)-\mathbf{Z}_{0}+\mathbf{U}(\mathbf{x})-\mathbf{\Delta}\right)}{\Theta\left(\mathcal{U}\left(\mathcal{P}_{\infty}^{+}\right)-\mathbf{Z}_{0}+\mathbf{U}(\mathbf{x})\right)} \exp \{2 \Phi(\mathbf{x})\}, \\
& q(\mathbf{x})=\frac{2 i \rho_{2} K_{0}^{2}}{A \rho_{1}} \frac{\Theta\left(\mathcal{U}\left(\mathcal{P}_{\infty}^{+}\right)-\mathbf{Z}_{0}+\mathbf{U}(\mathbf{x})+\boldsymbol{\Delta}\right)}{\Theta\left(\mathcal{U}\left(\mathcal{P}_{\infty}^{+}\right)-\mathbf{Z}_{0}+\mathbf{U}(\mathbf{x})\right)} \exp \{-2 \Phi(\mathbf{x})\},
\end{aligned}
$$

where

$$
A=\frac{\Theta\left(\mathcal{U}\left(\mathcal{P}_{\infty}^{+}\right)-\mathbf{Z}_{0}\right)}{\Theta\left(\mathcal{U}\left(\mathcal{P}_{\infty}^{-}\right)-\mathbf{Z}_{0}\right)}, \quad \Phi(\mathbf{x})=K_{1} x+\sum_{j \geqslant 1} K_{j+1} t_{j}
$$

\section{AlgEBRAiC-GEOMETRIC SOlutions CONSTRUCTED BY SPECTRAL CURVE WITH ANTI-INVOLUTION}

Let the canonical basis of cycles be transformed under the anti-holomorphic involution

$$
\tau_{a}:(\chi, \lambda) \rightarrow\left(\chi^{*}, \lambda^{*}\right)
$$

by the following formulae $\left(\sigma_{a}= \pm 1\right)$

$$
\tau_{a} \mathbf{a}=\sigma_{a} \mathbf{a}, \quad \tau_{a} \mathbf{b}=-\sigma_{a}(\mathbf{b}+K \mathbf{a}) .
$$


We introduce the notations

$$
\mathcal{A}_{j m}=\oint_{a_{j}} \lambda^{g-m} \frac{d \lambda}{\chi}, \quad \mathcal{B}_{j m}=\oint_{b_{j}} \lambda^{g-m} \frac{d \lambda}{\chi} .
$$

Then the matrix of the coefficients of the normalized holomorphic differentials (2.3) and matrix of periods 2.5 are

$$
\mathcal{C}=\left(\mathcal{A}^{t}\right)^{-1}, \quad B=\mathcal{B C}^{t}=\mathcal{B A}^{-1}
$$

The equation

$$
\int_{\tau \ell} d \omega=\int_{\ell} \tau d \omega
$$

where $\ell$ is an arbitrary path on $\Gamma$ and $d \omega$ is an arbitrary Abelian differential, implies that

$$
\begin{aligned}
\left(\mathcal{A}_{j m}\right)^{*} & =\oint_{a_{j}}\left(\lambda^{g-m} \frac{d \lambda}{\chi}\right)^{*}=\oint_{a_{j}} \tau_{a}\left(\lambda^{g-m} \frac{d \lambda}{\chi}\right) \\
& =\oint_{\tau_{a} a_{j}} \lambda^{g-m} \frac{d \lambda}{\chi}=\sigma_{a} \oint_{a_{j}} \lambda^{g-m} \frac{d \lambda}{\chi}=\sigma_{a} \mathcal{A}_{j m} .
\end{aligned}
$$

Therefore, $\mathcal{A}^{*}=\sigma_{a} \mathcal{A}$ and $\mathcal{C}^{*}=\sigma_{a} \mathcal{C}$. Proceeding in the same way with the integrals over $b$-cycles, we obtain

$$
\mathcal{B}^{*}=-\sigma_{a}(\mathcal{B}+K \mathcal{A}) \quad \text { or } \quad B^{*}=-B-K
$$

and

$$
\operatorname{Re}(B)=-\frac{1}{2} K
$$

Generalizing these formulae to an arbitrary path $\ell$, we have

$$
\left(\int_{\ell} d \mathcal{U}\right)^{*}=\sigma_{a} \int_{\tau_{a} \ell} d \mathcal{U}
$$

It follows from Riemann bilinear relations, see, for instance, [38], 46], 44], that

$$
V_{j}^{k}=\underset{\mathcal{P}_{\infty}^{+}}{\operatorname{Res}}\left(\mathcal{U}_{j}(\mathcal{P}) d \Omega_{k}\right)-\operatorname{Res}_{\mathcal{P}_{\infty}^{-}}\left(\mathcal{U}_{j}(\mathcal{P}) d \Omega_{k}\right)=\left.\frac{-2^{k} i^{k-1}}{(k-1) !} \frac{\partial^{k} \mathcal{U}_{j}}{\partial \xi_{+}^{k}}\right|_{\xi_{+}=0} .
$$

Hence,

$$
\left(\mathbf{V}^{k}\right)^{*}=(-1)^{k-1} \sigma_{a} \mathbf{V}^{k}
$$

The identities $\left(\Omega_{j}(\mathcal{P})\right)^{*}=(-1)^{j-1} \Omega_{j}\left(\tau_{a} \mathcal{P}\right)$ and $\tau_{a} \mathcal{P}_{\infty}^{ \pm}=\mathcal{P}_{\infty}^{ \pm}$yield that

$$
K_{j}^{*}=(-1)^{j-1} K_{j} \quad \text { and } \quad \Phi^{*}(\mathbf{x})=-\Phi(\widehat{J} \mathbf{x})
$$

where $\widehat{J}_{k m}=(-1)^{k} \delta_{k m}$.

We consider four types of spectral curves with involtuion 3.1 , 3.2).

1. All branching point do not lie on the real axis: $\operatorname{Im}\left(\lambda_{2 g+2}\right) \neq 0, \sigma_{a}=-1, \operatorname{Re}(B) \neq 0$ as $g>1$, see Figure 1 .

2. Only part of the branching points do not lie on the real axis, $\sigma_{a}=-1, \operatorname{Re}(B) \neq 0$ as $g>1$, see Figure 2.

3. All branching points lie on the real axis: $\operatorname{Im}\left(\lambda_{j}\right)=0, \sigma_{a}=-1, \operatorname{Re}(B)=0$, see Figure 3 .

4. All branching points lie on the real axis: $\operatorname{Im}\left(\lambda_{j}\right)=0, \sigma_{a}=1, \operatorname{Re}(B)=0$, see Figure 4 . In all four cases we assume that

$$
\Omega_{j}(\mathcal{P})=\int_{\mathcal{P}_{2 g+2}}^{\mathcal{P}} d \Omega_{j}, \quad \omega_{0}(\mathcal{P})=\int_{\mathcal{P}_{2 g+2}}^{\mathcal{P}} d \omega_{0}, \quad \mathcal{U}(\mathcal{P})=\int_{\mathcal{P}_{2 g+2}}^{\mathcal{P}} d \mathcal{U}
$$

Therefore, in all four cases, the following conditions are satisfied

$$
\Omega_{j}\left(\tau_{0} \mathcal{P}\right)=-\Omega_{j}(\mathcal{P}), \quad \omega_{0}\left(\tau_{0} \mathcal{P}\right)=-\omega_{0}(\mathcal{P}), \quad \mathcal{U}\left(\tau_{0} \mathcal{P}\right)=-\mathcal{U}(\mathcal{P})
$$




$$
\mathcal{U}\left(\mathcal{P}_{\infty}^{+}\right)=-\mathcal{U}\left(\mathcal{P}_{\infty}^{-}\right) \text {and } \quad \mathcal{U}\left(\mathcal{P}_{\infty}^{+}\right)=\frac{1}{2} \Delta
$$

where $\tau_{0}$ is a hyperelliptic involution, $\tau_{0}:(\chi, \lambda) \rightarrow(-\chi, \lambda)$.

In Case 1, the condition $\tau_{a} \mathcal{P}_{2 g+2}=\mathcal{P}_{2 g+1}$ holds. This is why by equation (3.4) and the relation $\tau_{a} \mathcal{P}_{\infty}^{ \pm}=\mathcal{P}_{\infty}^{ \pm}$we obtain the following identities

$$
\begin{aligned}
& \left(\mathcal{U}\left(\mathcal{P}_{\infty}^{+}\right)\right)^{*}=\left(\int_{\mathcal{P}_{2 g+2}}^{\mathcal{P}_{\infty}^{+}} d \mathcal{U}\right)^{*}=\sigma_{a} \int_{\mathcal{P}_{2 g+1}}^{\mathcal{P}_{\infty}^{+}} d \mathcal{U}=\sigma_{a} \int_{\mathcal{P}_{2 g+1}}^{\mathcal{P}_{2 g+2}} d \mathcal{U}+\sigma_{a} \mathcal{U}\left(\mathcal{P}_{\infty}^{+}\right) \\
& \left(\mathcal{U}\left(\mathcal{P}_{\infty}^{-}\right)\right)^{*}=\left(\int_{\mathcal{P}_{2 g+2}}^{\mathcal{P}_{\infty}^{-}} d \mathcal{U}\right)^{*}=\sigma_{a} \int_{\mathcal{P}_{2 g+1}}^{\mathcal{P}_{\infty}^{-}} d \mathcal{U}=\sigma_{a} \int_{\mathcal{P}_{2 g+1}}^{\mathcal{P}_{2 g+2}} d \mathcal{U}+\sigma_{a} \mathcal{U}\left(\mathcal{P}_{\infty}^{-}\right)
\end{aligned}
$$

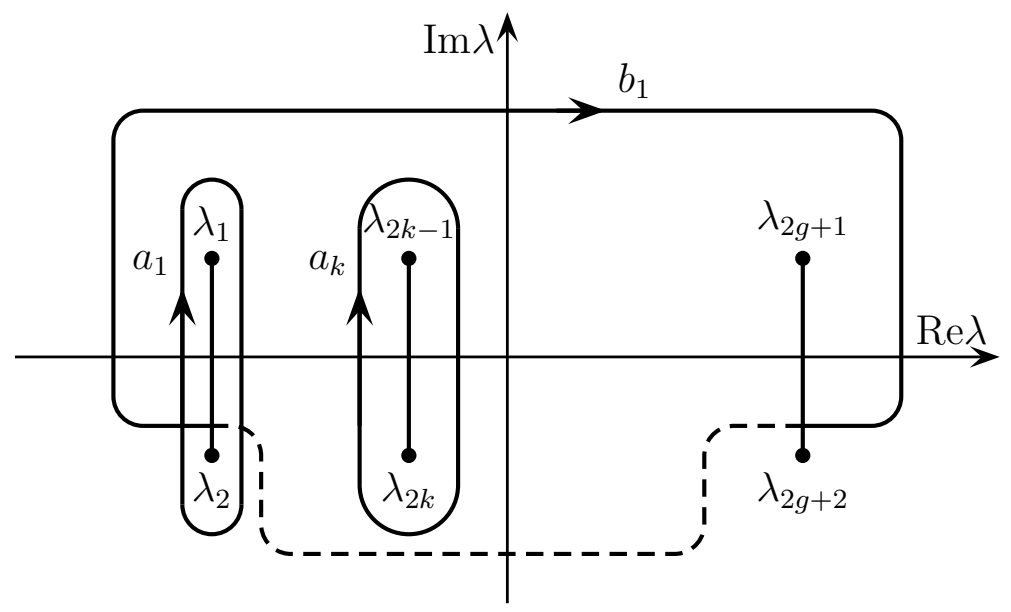

FiguRE 1. Case 1

We observe that the integration paths in equations $(3.6)$ belong to two different sheets of two-sheet surface $\Gamma$. This is why, in Case 1

$$
\Delta^{*}=-\sigma_{a} \sum_{k=1}^{g} \int_{a_{k}} d \mathcal{U}+\sigma_{a} \Delta=\mathbf{e}-\Delta \quad \text { or } \quad \operatorname{Re}(\Delta)=\frac{1}{2} \mathbf{e},
$$

where $e_{j}=1, j=1, \ldots, g$.

Also in this case the identity

$$
\left(\omega_{0}(\mathcal{P})\right)^{*}=\left(\int_{\mathcal{P}_{2 g+2}}^{\mathcal{P}} d \omega_{0}\right)^{*}=\int_{\mathcal{P}_{2 g+2}}^{\mathcal{P}} \tau_{a}\left(d \omega_{0}\right)=\int_{\mathcal{P}_{2 g+1}}^{\tau_{a} \mathcal{P}} d \omega_{0}=\int_{\mathcal{P}_{2 g+1}}^{\mathcal{P}_{2 g+2}} d \omega_{0}+\omega_{0}\left(\tau_{a} \mathcal{P}\right),
$$

holds, where the integration path connecting the points $\mathcal{P}_{2 g+1}$ and $\mathcal{P}_{2 g+2}$ do not intersect the basis cycles. Calculating the integral $\int_{\mathcal{P}_{2 g+1}}^{\mathcal{P}_{2 g+2}} d \omega_{0}$, we obtain

$$
\int_{\mathcal{P}_{2 g+1}}^{\mathcal{P}_{2 g+2}} d \omega_{0}=\frac{1}{2}\left(\sum_{k=1}^{g} \int_{a_{k}} d \omega_{0}+\underset{\mathcal{P}_{\infty}^{+}}{2 \pi i \operatorname{Res}}\left(d \omega_{0}\right)\right)=-\pi i .
$$

Therefore,

$$
\begin{aligned}
\operatorname{Im}\left(\ln K_{0}\right) & =\frac{1}{2 i} \lim _{\mathcal{P} \rightarrow \mathcal{P}_{\infty}^{+}}\left(\omega_{0}(\mathcal{P})-\left(\omega_{0}(\mathcal{P})\right)^{*}\right) \\
& =\frac{\pi}{2}+\frac{1}{2 i} \lim _{\mathcal{P} \rightarrow \mathcal{P}_{\infty}^{+}} \int_{\tau_{a} \mathcal{P}}^{\mathcal{P}} d \omega_{0}=\frac{\pi}{2}+\pi n, \quad n \in\{0 ; 1 ;-1\}
\end{aligned}
$$


or $K_{0}^{2}=-\left|K_{0}\right|^{2}$.

Choosing the initial phase $\mathbf{Z}_{0}$ so that it satisfies the condition

$$
\left(\mathcal{U}\left(\mathcal{P}_{\infty}^{+}\right)-\mathbf{Z}_{0}\right)^{*}=\mathcal{U}\left(\mathcal{P}_{\infty}^{+}\right)-\mathbf{Z}_{0}+B \mathbf{M}+\mathbf{N}, \quad \mathbf{M}, \mathbf{N} \in \mathbb{Z}^{g}, \quad \mathbf{N}=-(\operatorname{Re} B) \mathbf{M},
$$

we get:

$$
\begin{aligned}
p^{*}(\mathbf{x}) & =\frac{-2 i A^{*} \rho_{1}^{*}}{\rho_{2}^{*}} \frac{\Theta\left(\left(\mathcal{U}\left(\mathcal{P}_{\infty}^{+}\right)-\mathbf{Z}_{0}\right)^{*}+U(\widehat{J} \mathbf{x})+\Delta-\mathbf{e}\right)}{\Theta\left(\left(\mathcal{U}\left(\mathcal{P}_{\infty}^{+}\right)-\mathbf{Z}_{0}\right)^{*}+U(\widehat{J} \mathbf{x})\right)} e^{-2 \Phi\left(\widehat{J}_{\mathbf{x}}\right)} \\
& =-e^{\pi i \mathbf{M}^{t} \mathbf{e}}\left|\frac{A \rho_{1}}{K_{0} \rho_{2}}\right|^{2} e^{2 \pi \mathbf{M}^{t} \operatorname{Im} \Delta} q(\widehat{J} \mathbf{x})
\end{aligned}
$$

Thus, as $\left|\rho_{2}\right|=\left|A \rho_{1} K_{0}^{-1} \exp \left\{\pi \mathbf{M}^{t} \operatorname{Im} \Delta\right\}\right|$, functions 2.10 constructed by the hyperelliptic curve possessing involution (3.1), 3.2 and satisfying the conditions $\operatorname{Im}\left(\lambda_{j}\right) \neq 0, \sigma_{a}=-1$, are algebraic-geometric solutions to nonlocal equations in AKNS hierarchy with the reduction $q(\mathbf{x})=\sigma p^{*}(\widehat{J} \mathbf{x})$, where

$$
\sigma=-\exp \left\{\pi i \mathbf{M}^{t} \mathbf{e}\right\}
$$

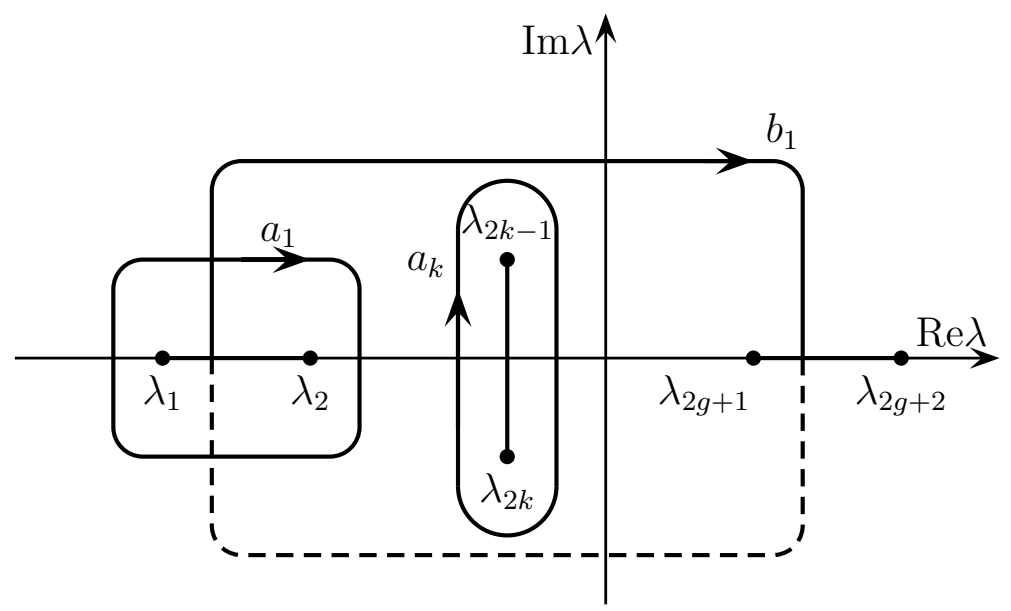

Figure 2. Case 2

In Case 2 there are non-zero diagonal entries in the matrix $K$. By the formula

$$
\Theta^{*}(p \mid B)=\Theta\left(p^{*}+\mathbf{d} \mid B\right),
$$

where $(\mathbf{d})_{j}=K_{j j} / 2$, we conclude that the spectral curve of this type can not be employed for constructing nonlocal reductions of multi-phase solutions in AKNS hierarchy.

In Case 3 the condition $\tau_{a} \mathcal{P}_{2 g+2}=\mathcal{P}_{2 g+2}$ holds and this is why

$$
\left(\mathcal{U}\left(\mathcal{P}_{\infty}^{+}\right)\right)^{*}=-\mathcal{U}\left(\mathcal{P}_{\infty}^{+}\right), \quad\left(\mathcal{U}\left(\mathcal{P}_{\infty}^{-}\right)\right)^{*}=-\mathcal{U}\left(\mathcal{P}_{\infty}^{-}\right) \quad \text { and } \quad \Delta^{*}=-\Delta
$$

and

Therefore,

$$
\left(\omega_{0}(\mathcal{P})\right)^{*}=\left(\int_{\mathcal{P}_{2 g+2}}^{\mathcal{P}} d \omega_{0}\right)^{*}=\int_{\mathcal{P}_{2 g+2}}^{\mathcal{P}} \tau_{a}\left(d \omega_{0}\right)=\int_{\mathcal{P}_{2 g+2}}^{\tau_{a} \mathcal{P}} d \omega_{0}=\omega_{0}\left(\tau_{a} \mathcal{P}\right)
$$

$$
\operatorname{Im}\left(\ln K_{0}\right)=\frac{1}{2 i} \lim _{\mathcal{P} \rightarrow \mathcal{P}_{\infty}^{+}}\left(\omega_{0}(\mathcal{P})-\left(\omega_{0}(\mathcal{P})\right)^{*}\right)=\frac{1}{2 i} \lim _{\mathcal{P} \rightarrow \mathcal{P}_{\infty}^{+}} \int_{\tau_{a} \mathcal{P}}^{\mathcal{P}} d \omega_{0}=\pi n, \quad n \in\{0 ; 1 ;-1\}
$$

or $K_{0}^{2}=\left|K_{0}\right|^{2}$.

Choosing the initial phase $\mathbf{Z}_{0}$ so that it satisfies the condition

$$
\left(\mathcal{U}\left(\mathcal{P}_{\infty}^{+}\right)-\mathbf{Z}_{0}\right)^{*}=\mathcal{U}\left(\mathcal{P}_{\infty}^{+}\right)-\mathbf{Z}_{0}+B \mathbf{M}, \quad \mathbf{M} \in \mathbb{Z}^{g},
$$




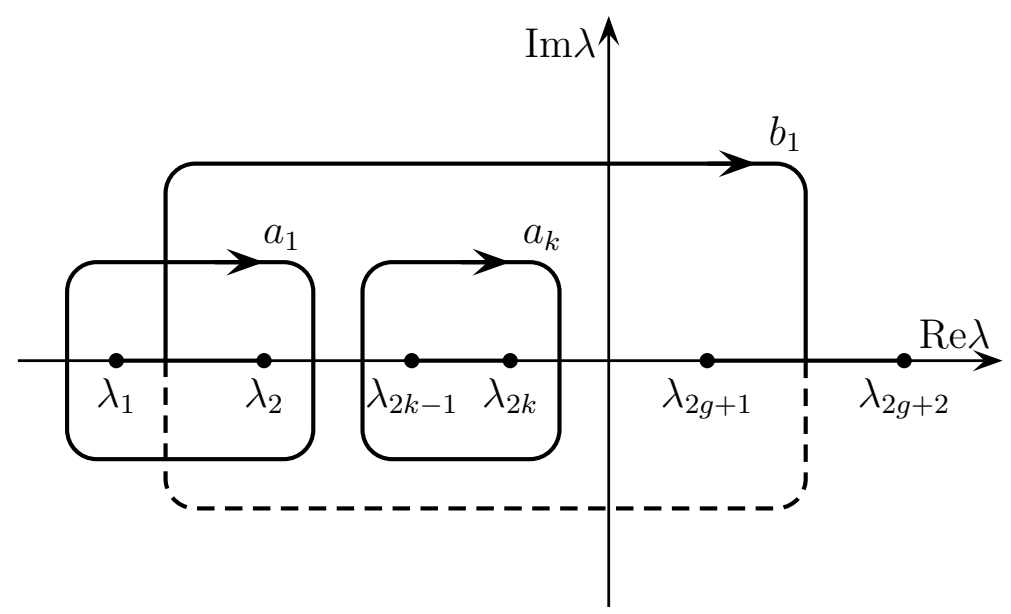

Figure 3. Case 3

we find:

$$
\begin{aligned}
p^{*}(\mathbf{x}) & =\frac{-2 i A^{*} \rho_{1}^{*}}{\rho_{2}^{*}} \frac{\Theta\left(\left(\mathcal{U}\left(\mathcal{P}_{\infty}^{+}\right)-\mathbf{Z}_{0}\right)^{*}+U(\widehat{J} \mathbf{x})+\Delta\right)}{\Theta\left(\left(\mathcal{U}\left(\mathcal{P}_{\infty}^{+}\right)-\mathbf{Z}_{0}\right)^{*}+U(\widehat{J} \mathbf{x})\right)} e^{-2 \Phi(\widehat{J} \mathbf{x})} \\
& =\left|\frac{A \rho_{1}}{K_{0} \rho_{2}}\right|^{2} e^{2 \pi \mathbf{M}^{t} \operatorname{Im} \Delta} q(\widehat{J} \mathbf{x})
\end{aligned}
$$

Thus, as $\left|\rho_{2}\right|=\left|A \rho_{1} K_{0}^{-1} \exp \left\{\pi \mathbf{M}^{t} \operatorname{Im} \Delta\right\}\right|$, functions 2.10 constructed by the hyperelliptic curve possessing involution (3.1), (3.2) and satisfying conditions $\operatorname{Re}(B)=0, \operatorname{Im}\left(\lambda_{j}\right)=0$, are algebraic-geometric solutions to nonlocal equations in AKNS hierarchy with the reduction $q(\mathbf{x})=p^{*}(\widehat{J} \mathbf{x})$.

For Case 4, the condition $\tau_{a} \mathcal{P}_{2 g+2}=\mathcal{P}_{2 g+2}$ holds again. However, since $\sigma_{a}=1$, we have

$$
\left(\mathcal{U}\left(\mathcal{P}_{\infty}^{+}\right)\right)^{*}=\mathcal{U}\left(\mathcal{P}_{\infty}^{+}\right), \quad\left(\mathcal{U}\left(\mathcal{P}_{\infty}^{-}\right)\right)^{*}=\mathcal{U}\left(\mathcal{P}_{\infty}^{-}\right) \quad \text { and } \quad \Delta^{*}=\Delta
$$

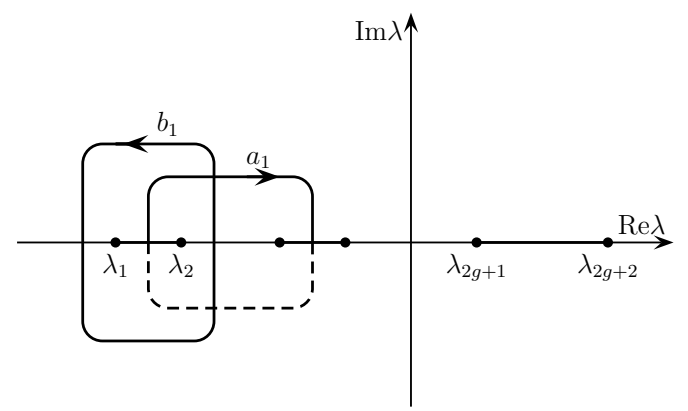

Figure 4. Case 4

Similar to Case 3, the identities hold:

$$
\left(\omega_{0}(\mathcal{P})\right)^{*}=\left(\int_{\mathcal{P}_{2 g+2}}^{\mathcal{P}} d \omega_{0}\right)^{*}=\int_{\mathcal{P}_{2 g+2}}^{\mathcal{P}} \tau_{a}\left(d \omega_{0}\right)=\int_{\mathcal{P}_{2 g+2}}^{\tau_{a} \mathcal{P}} d \omega_{0}=\omega_{0}\left(\tau_{a} \mathcal{P}\right)
$$

and

$$
\operatorname{Im}\left(\ln K_{0}\right)=\frac{1}{2 i} \lim _{\mathcal{P} \rightarrow \mathcal{P}_{\infty}^{+}}\left(\omega_{0}(\mathcal{P})-\left(\omega_{0}(\mathcal{P})\right)^{*}\right)=\frac{1}{2 i} \lim _{\mathcal{P} \rightarrow \mathcal{P}_{\infty}^{+}} \int_{\tau_{a} \mathcal{P}}^{\mathcal{P}} d \omega_{0}=\pi n, \quad n \in\{0 ; 1 ;-1\}
$$

or $K_{0}^{2}=\left|K_{0}\right|^{2}$. 
Choosing the initial phase $\mathbf{Z}_{0}$ so that it satisfies the condition

$$
\left(\mathcal{U}\left(\mathcal{P}_{\infty}^{+}\right)-\mathbf{Z}_{0}\right)^{*}=-\left(\mathcal{U}\left(\mathcal{P}_{\infty}^{+}\right)-\mathbf{Z}_{0}\right)+\mathbf{N}, \quad \mathbf{N} \in \mathbb{Z}^{g},
$$

we obtain:

$$
p^{*}(\mathbf{x})=\frac{-2 i A^{*} \rho_{1}^{*}}{\rho_{2}^{*}} \frac{\Theta\left(\mathbf{N}-\left(\mathcal{U}\left(\mathcal{P}_{\infty}^{+}\right)-\mathbf{Z}_{0}+U(\widehat{J} \mathbf{x})+\Delta\right)\right)}{\Theta\left(\mathbf{N}-\left(\mathcal{U}\left(\mathcal{P}_{\infty}^{+}\right)-\mathbf{Z}_{0}+U(\widehat{J} \mathbf{x})\right)\right)} e^{-2 \Phi(\widehat{J} \mathbf{x})}=\left|\frac{A \rho_{1}}{K_{0} \rho_{2}}\right|^{2} q(\widehat{J} \mathbf{x}) .
$$

Thus, as $\left|\rho_{2}\right|=\left|A \rho_{1} K_{0}^{-1}\right|$ functions 2.10 constructed by the hyperelliptic curve possessing involution (3.1), (3.2) and satisfying the conditions $\operatorname{Im}\left(\lambda_{2 g+2}\right)=0$, are algebraic-geometric solutions to nonlocal equations in AKNS hierarchy with the reduction $q(\mathbf{x})=p^{*}(\widehat{J} \mathbf{x})$.

\section{Solutions CONSTRUCTED SPECTRAL CURVE With HOLOMORPHIC INVOLUTiON}

Unfortunately, as $K_{2 j-1} \neq 0$, algebraic-geometric solutions of $(2.10)$ constructed by the hyperelliptic curve possessing involution (3.1), (3.2) (3.1), (3.2) grow exponentially with respect to the corresponding variables. This can be avoided if we employ hyperelliptic curves with the holomorphic involution

$$
\tau_{h}:(\chi, \lambda) \rightarrow(\chi,-\lambda)
$$

It is easy to see that in all four considered cases, the bases of cycles are transformed by the rule

$$
\tau_{h} \mathbf{a}=S \mathbf{a}, \quad \tau_{h} \mathbf{b}=Q \mathbf{a}+R \mathbf{b}, \quad \tau_{h} \mathcal{P}_{\infty}^{ \pm}=\mathcal{P}_{\infty}^{ \pm},
$$

where, see, for instance, [38], [52],

$$
S R^{t}=I \quad \text { and } \quad Q R^{t}=R Q^{t} .
$$

Calculating the periods of holomorphic differentials $d \widehat{\mathcal{U}}_{j}(\mathcal{P})=d \mathcal{U}_{j}\left(\tau_{h} \mathcal{P}\right)$, we obtain:

$$
\begin{aligned}
\int_{a_{k}} d \widehat{\mathcal{U}}_{j}(\mathcal{P}) & =\int_{\tau_{h} a_{k}} d \mathcal{U}_{j}=\sum_{m=1}^{g} S_{k m} \int_{a_{m}} d \mathcal{U}_{j}=S_{k j}, \\
\int_{a_{k}} d \widehat{\mathcal{U}}_{j}(\mathcal{P}) & =\sum_{m=1}^{g} c_{j m} \int_{a_{k}} \tau_{h}\left(\lambda^{g-m} \frac{d \lambda}{\chi}\right)=\sum_{m=1}^{g} c_{j m}(-1)^{g+1-m} \mathcal{A}_{k m}=\left(\mathcal{A} J \mathcal{C}^{t}\right)_{k j},
\end{aligned}
$$

where

$$
J_{m n}=(-1)^{g+1-m} \delta_{m n}
$$

Therefore, $d \widehat{\mathcal{U}}=S^{t} d \mathcal{U}$, and the matrices $S$ and $J$ are similar:

$$
S=\left(\mathcal{C}^{t}\right)^{-1} J \mathcal{C}^{t} \quad \text { and } \quad S^{t}=\mathcal{C} J \mathcal{C}^{-1} .
$$

Since $R=\left(S^{t}\right)^{-1}$ and $S^{2}=I$, then $R=S^{t}$.

We integrate holomorphic differentials over $b$-cycles:

$$
\begin{aligned}
\int_{b_{k}} d \widehat{\mathcal{U}}_{j}(\mathcal{P}) & =\int_{\tau_{h} b_{k}} d \mathcal{U}_{j}=\sum_{m=1}^{g}\left(Q_{k m} \int_{a_{m}} d \mathcal{U}_{j}+R_{k m} \int_{b_{m}} d \mathcal{U}_{j}\right)=(Q+R B)_{k j} \\
\int_{b_{k}} d \widehat{\mathcal{U}}_{j}(\mathcal{P}) & =\sum_{m=1}^{g}\left(S^{t}\right)_{j m} \int_{b_{k}} d \mathcal{U}_{m}=(B S)_{k j},
\end{aligned}
$$

or

$$
B S=Q+R B
$$

We transpose identity 4.3 and we find:

$$
S^{t} B=Q^{t}+B R^{t} \quad \text { or } \quad R B=Q^{t}+B S .
$$

Therefore, $Q^{t}=-Q$. Calculating the real part of identity (4.3), we get:

$$
Q=(\operatorname{Re} B) S-S^{t}(\operatorname{Re} B)
$$


and

$$
S^{t} Q=S^{t}(\operatorname{Re} B) S-(\operatorname{Re} B)=Q^{t} S .
$$

We observe that it follows from the asymptotics of the function $\chi(\lambda)$ in the vicinity of the infinite points that

- if $g$ is odd, then $\tau_{h} \mathcal{P}_{\infty}^{ \pm}=\mathcal{P}_{\infty}^{ \pm}$,

- if $g$ is even, then $\tau_{h} \mathcal{P}_{\infty}^{ \pm}=\mathcal{P}_{\infty}^{\mp}$.

Therefore,

$$
\begin{aligned}
S^{t} \Delta & =2 \int_{\mathcal{P}_{2 g+2}}^{\mathcal{P}_{\infty}^{+}} S^{t} d \mathcal{U}=2 \int_{\mathcal{P}_{2 g+2}}^{\mathcal{P}_{\infty}^{+}} \tau_{h} d \mathcal{U}=2 \int_{\mathcal{P}_{1}}^{\tau_{h} \mathcal{P}_{\infty}^{+}} d \mathcal{U} \\
& =2 \int_{\mathcal{P}_{1}}^{\mathcal{P}_{2 g+2}} d \mathcal{U}+2 \mathcal{U}\left(\tau_{h} \mathcal{P}_{\infty}^{+}\right)=(-1)^{g+1} \Delta-2 \mathcal{U}\left(\mathcal{P}_{1}\right)
\end{aligned}
$$

We introduce the notations: $\widehat{\Omega}_{j}(\mathcal{P})=\Omega_{j}\left(\tau_{h} \mathcal{P}\right)$. These integrals possess the following properties:

$$
\begin{aligned}
& \int_{a_{k}} d \widehat{\Omega}_{j}=\int_{\tau_{h} a_{k}} d \Omega_{j}=\sum_{m=1}^{g} S_{k m} \int_{a_{m}} d \Omega_{j}=0, \\
& \widehat{\Omega}_{j}(\mathcal{P})= \pm\left((2 i)^{j-1}(-\lambda)^{j}-K_{j}+O\left(\lambda^{-1}\right)\right), \quad \mathcal{P} \rightarrow \mathcal{P}_{\infty}^{ \pm} .
\end{aligned}
$$

Since the Abelian integral $\mu_{j}(\mathcal{P})=\widehat{\Omega}_{j}(\mathcal{P})-(-1)^{j} \Omega_{j}(\mathcal{P})$ possesses zero $a$-periods and no singular singularities, it is constant. The asymptotics of $\mu_{j}(\mathcal{P})$ at infinite points

$$
\mu_{j}(\mathcal{P})=\mp\left((-1)^{j}-1\right) K_{j}+O\left(\lambda^{-1}\right), \quad \mathcal{P} \rightarrow \mathcal{P}_{\infty}^{ \pm}
$$

implies that $\mu_{j}(\mathcal{P}) \equiv 0, \widehat{\Omega}_{j}(\mathcal{P}) \equiv(-1)^{j} \Omega_{j}(\mathcal{P}), K_{2 j-1}=0$.

Thus, multi-phase solutions 2.10 constructed by hyperelliptic curve 2.2 with involutions (3.1), 4.1) has no exponential growth.

Calculating $b$-periods of Abelian integrals of second kind, we obtain:

$$
\begin{aligned}
\left(\widehat{\mathbf{V}}^{j}\right)_{k} & =\frac{1}{2 \pi i} \int_{b_{k}} d \widehat{\Omega}_{j}=\frac{1}{2 \pi i} \int_{\tau_{h} b_{k}} d \Omega_{j} \\
& =\frac{1}{2 \pi i} \sum_{m=1}^{g}\left(Q_{k m} \int_{a_{m}} d \Omega_{j}+R_{k m} \int_{b_{m}} d \Omega_{j}\right)=\left(R \mathbf{V}^{j}\right)_{k}, \\
\left(\widehat{\mathbf{V}}^{j}\right)_{k} & =\frac{1}{2 \pi i} \int_{b_{k}} d \widehat{\Omega}_{j}=\frac{(-1)^{j}}{2 \pi i} \int_{b_{k}} d \Omega_{j}=(-1)^{j}\left(\mathbf{V}^{j}\right)_{k} .
\end{aligned}
$$

Therefore, the vectors $\mathbf{V}^{j}$ are the eigenvectors of the matrix $R: R \mathbf{V}^{j}=(-1)^{j} \mathbf{V}^{j}$ or

$$
S^{t} \mathbf{V}^{j}=(-1)^{j} \mathbf{V}^{j}
$$

We considered single-phase solutions of nonlocal equations in AKNS hierarchy in works [31][33. This is why in what follows we assume that $g>1$.

In Case 1 the identity $\operatorname{Re}\left(B_{j k}\right)=\left(\delta_{j k}-1\right) / 2$ holds, and the entries of the matrices of cycles transformation under the involution $\tau_{h}$ are

$$
\begin{aligned}
& S_{1 k}=(-1)^{g}, \quad S_{j k}=(-1)^{g+1} \delta_{j, g+2-k}, \quad j=2, \ldots, g, \quad k=1, \ldots, g, \\
& Q_{j k}=(-1)^{g}\left(\delta_{j 1}-\delta_{1 k}\right), \quad j, k=1, \ldots, g, \\
& \left(S^{t} Q\right)_{j k}=1-\delta_{j 1} \delta_{1 k} .
\end{aligned}
$$

In particular, as $g=2$,

$$
S=\left(\begin{array}{cc}
1 & 1 \\
0 & -1
\end{array}\right), \quad Q=\left(\begin{array}{cc}
0 & 1 \\
-1 & 0
\end{array}\right), \quad \operatorname{Re} B=-\frac{1}{2}\left(\begin{array}{ll}
0 & 1 \\
1 & 0
\end{array}\right),
$$


while for $g=3$ we have

$$
S=\left(\begin{array}{ccc}
-1 & -1 & -1 \\
0 & 0 & 1 \\
0 & 1 & 0
\end{array}\right), \quad Q=\left(\begin{array}{ccc}
0 & -1 & -1 \\
1 & 0 & 0 \\
1 & 0 & 0
\end{array}\right), \quad \operatorname{Re} B=-\frac{1}{2}\left(\begin{array}{ccc}
0 & 1 & 1 \\
1 & 0 & 1 \\
1 & 1 & 0
\end{array}\right)
$$

It follows from equations (4.4), (4.6) and the realness of the vector $\Delta$ that

$$
S^{t} \Delta=(-1)^{g+1}\left(\Delta+(B-I) \mathbf{e}_{1}\right),
$$

where $\mathbf{e}_{1}^{t}=(1,0, \ldots, 0)$.

It can be shown that for even $g$, condition (3.7) is satisfied only for $\mathbf{M} \in 2 \mathbb{Z}^{g}$. Therefore, for even $g$, solution (2.10) constructed by curve (2.2), (3.1), (4.1), satisfies the reduction

$$
q(\mathbf{x})=-p^{*}(\widehat{J} \mathbf{x})
$$

At the same time, for odd $g$, there exist initial phases $\mathbf{Z}_{0}$ for the reductions of both types:

$$
q(\mathbf{x})= \pm p^{*}(\widehat{J} \mathbf{x})
$$

In Case 3, the entries of the matrix $S$ are determined by formula (4.6) and the identities hold:

$$
\operatorname{Re}\left(B_{j k}\right)=0, \quad Q_{j k}=0, \quad S^{t} \Delta=(-1)^{g+1}\left(\Delta+B \mathbf{e}_{1}\right) .
$$

It is easy to show that in Case 3 , for each $\mathbf{M} \in \mathbb{Z}^{g}$ and for an initial phase $\mathbf{Z}_{0}$ obeying condition (3.9), solution $(2.10)$ constructed by curve (2.2), (3.1), (4.1) satisfies the reduction

$$
q(\mathbf{x})=p^{*}\left(\widehat{J}_{\mathbf{x}}\right)
$$

\section{In Case 4}

$$
\begin{aligned}
& \operatorname{Re}\left(B_{j k}\right)=0, \quad Q_{j k}=0, \quad S_{j k}=(-1)^{g} \delta_{j, g+1-k}, \quad j, k=1, \ldots, g, \\
& S^{t} \Delta=(-1)^{g+1}(\Delta-\mathbf{e}),
\end{aligned}
$$

and for each $\mathbf{N} \in \mathbb{Z}^{g}$, with an initial phase $\mathbf{Z}_{0}$ obeying condition 3.10 , solution $(2.10)$ constructed by curve (2.2), (3.1), (4.1) satisfies the reduction

$$
q(\mathbf{x})=p^{*}(\widehat{J} \mathbf{x})
$$

\section{Reduction of ALGEBRAIC-GEOMEtRiC SOlution to THETA FUNCTIONS OF LOWER DIMENSION}

5.1. General facts. It follows from equation (4.3) that the matrix of periods $B$ satisfies the equation

$$
B=S^{t} B S-S^{t} Q
$$

Following [52, we consider a matrix $T, T_{j k} \in \mathbb{Z}$, obeying the condition

$$
S=T J T^{-1},
$$

where the matrix $J$ is determined by formula 4.2 .

In the first and third cases, when the matrix $S$ is determined by conditions (4.6), equation (5.2) implies the following requirements for the entries of the matrix $T$ :

$$
\begin{aligned}
& \sum_{m=2}^{g} T_{m k}=\left((-1)^{k-1}-1\right) T_{1 k}, \\
& T_{g+2-j, k}=(-1)^{k} T_{j k}, \quad j=2, \ldots, g, \quad k=1, \ldots, g .
\end{aligned}
$$

We fix the entries in the first row of the matrix $T$ :

$$
T_{1 k}=1, \quad k=1, \ldots, g .
$$


We define other entries of the matrix $T$ as follows. If $g=2 m, m \in \mathbb{N}$, then

$$
\begin{aligned}
& T_{j, 2 k}=-\delta_{j, m+k}-\delta_{j, m+2-k}, \\
& T_{j, 2 k-1}=\delta_{j, m+k}-\delta_{j, m+2-k}, \quad j=2, \ldots, g, \quad k=1, \ldots, m .
\end{aligned}
$$

If $g=2 m+1, m \in \mathbb{N}$, then $T_{j 1}=0$,

$$
\begin{aligned}
& T_{j, 2 k}=-\delta_{j, m+1+k}-\delta_{j, m+2-k}, \\
& T_{j, 2 k+1}=\delta_{j, m+1+k}-\delta_{j, m+2-k}, \quad j=2, \ldots, g, \quad k=1, \ldots, m .
\end{aligned}
$$

The properties of determinants imply that $\operatorname{det} T=(-2)^{m}$.

In the fourth case the requirements for the entries of the matrix $T$ are of the form

$$
T_{g+1-j, k}=(-1)^{k-1} T_{j k} .
$$

We define the entries of the matrix $T$ as follows. If $g=2 m, m \in \mathbb{N}$, then

$$
\begin{aligned}
& T_{j k}=1 \quad \text { as } \quad 1 \leqslant j \leqslant m, \quad 1 \leqslant k \leqslant 2(m+1-j), \\
& T_{j k}=0 \quad \text { as } \quad 1<j \leqslant m, \quad 2(m+1-j)<k \leqslant 2 m, \quad m \neq 1, \\
& T_{j k}=(-1)^{k-1} \quad \text { as } \quad m<j \leqslant 2 m, \quad 1 \leqslant k \leqslant 2(j-m), \\
& T_{j k}=0 \quad \text { as } \quad m<j<2 m, \quad 2(j-m)<k \leqslant 2 m, \quad m \neq 1 .
\end{aligned}
$$

If $g=2 m+1, m \in \mathbb{N}$, then

$$
\begin{aligned}
& T_{j k}=1 \quad \text { as } \quad 1 \leqslant j \leqslant m, \quad 1 \leqslant k \leqslant 2(m-j)+3, \\
& T_{j k}=0 \quad \text { as } \quad 1<j \leqslant m, \quad 2(m-j)+3<k \leqslant 2 m+1, \quad m \neq 1, \\
& T_{j k}=(-1)^{k-1} \quad \text { as } \quad m<j \leqslant 2 m+1, \quad 1 \leqslant k \leqslant 2(j-m)-1, \\
& T_{j k}=0 \quad \text { as } \quad m<j<2 m+1, \quad 2(j-m) \leqslant k \leqslant 2 m+1 .
\end{aligned}
$$

In the case the identity $\operatorname{det} T=(-2)^{m}$ holds as well.

We introduce the notations:

$$
\widetilde{B}=T^{t}(i \operatorname{Im} B) T, \quad \widetilde{A}=T^{t}(\operatorname{Re} B) T, \quad \widetilde{\mathbf{V}}^{j}=T^{t} \mathbf{V}^{j} .
$$

Equations (4.5), (5.1) and (5.2) imply the following relations

$$
\widetilde{B}=J \widetilde{B} J, \quad J \widetilde{\mathbf{V}}^{j}=(-1)^{j} \widetilde{\mathbf{V}}^{j}, \quad(\widetilde{A})_{j k} \in \mathbb{Z} .
$$

Changing the summation order in the formula for the multi-dimensional theta function with the matrix $B$, we obtain (see $52 \mid$ )

$$
\Theta(\mathbf{p} \mid B)=\sum_{\mathbf{k} \in \mathbb{Z}^{g}(T)} e^{-\pi i \boldsymbol{\eta}^{t}(\mathbf{k})(\widetilde{A}-D) \boldsymbol{\eta}(\mathbf{k})} \Theta\left[\boldsymbol{\eta}^{t}(\mathbf{k}) ; \boldsymbol{\zeta}^{t}(\mathbf{k})\right]\left(T^{t} \mathbf{p} \mid \widetilde{B}+D\right),
$$

where the summation $\mathbf{k} \in \mathbb{Z}^{g}(T)$ denotes a finite sum over $\mathbf{k}: \mathbf{k} \in \mathbb{Z}^{g}, 0 \leqslant T^{-1} \mathbf{k}<1, D$ is a diagonal matrix, $D_{j j}=\widetilde{A}_{j j}, \boldsymbol{\eta}(\mathbf{k})=T^{-1} \mathbf{k}, \boldsymbol{\zeta}(\mathbf{k})=(\widetilde{A}-D) \boldsymbol{\eta}(k)$. The number of the terms in the sum is equal to $|\operatorname{det} T|$. At that, since relations 5.3 hold, the matrix $\widetilde{B}$ has a block structure and the theta function

$$
\Theta\left[\boldsymbol{\eta}^{t}(\mathbf{k}) ; \boldsymbol{\zeta}^{t}(\mathbf{k})\right]\left(T^{t} \mathbf{p} \mid \widetilde{B}+D\right)
$$

can be represented as the product of two theta functions of lower dimension. It also follows from relations (5.3) that one of theta functions depends on times with odd indices $t_{1}, t_{3}, \ldots$, while the does on the variable $x$ and the times with even indices $t_{2}, t_{4}, \ldots$

In conclusion of this section we provide examples of representing two-phase solutions for nonlocal equations in AKNS hierarchy via one-dimensional theta functions. 
5.2. Two-phase solution. Case 1. The calculations made for the spectral curve

$$
\chi^{2}=\left(\lambda^{2}+c^{2}\right)\left(\lambda^{4}-2\left(a^{2}-b^{2}\right) \lambda^{2}+\left(a^{2}+b^{2}\right)^{2}\right), \quad a, b, c \in \mathbb{R},
$$

give the following formulae:

$$
\begin{gathered}
B=\left(\begin{array}{cc}
2 i \beta_{1} & i \beta_{1}-1 / 2 \\
i \beta_{1}-1 / 2 & i \beta_{2}
\end{array}\right), \quad \Delta=\left(\begin{array}{c}
1 / 2-i \beta_{1} \\
1 / 2+i \delta_{2}
\end{array}\right), \\
\mathbf{V}^{2 j-1}=\left(\begin{array}{c}
0 \\
i v_{2 j-1}
\end{array}\right), \quad \mathbf{V}^{2 j}=\left(\begin{array}{c}
2 v_{2 j} \\
v_{2 j}
\end{array}\right),
\end{gathered}
$$

where $\beta_{j}, \delta_{j}, v_{j} \in \mathbb{R}$. Therefore,

$$
\begin{array}{cc}
\widetilde{B}=\left(\begin{array}{cc}
2 i \beta_{1} & 0 \\
0 & 4 i \beta_{2}-2 i \beta_{1}
\end{array}\right), & \widetilde{A}=\left(\begin{array}{ll}
0 & 1 \\
1 & 2
\end{array}\right), \\
\widetilde{\mathbf{V}}^{2 j-1}=\left(\begin{array}{c}
0 \\
-2 i v_{2 j-1}
\end{array}\right), & \widetilde{\mathbf{V}}^{2 j}=\left(\begin{array}{c}
2 v_{2 j} \\
0
\end{array}\right) .
\end{array}
$$

It is easy to confirm that the corresponding two-dimensional theta function

$$
\begin{aligned}
f_{s}(\mathbf{x}) & =\Theta\left(\mathbf{Z}+\mathbf{V}^{1} x+\sum_{j \geqslant 1} \mathbf{V}^{j+1} t_{j}+s \Delta \mid B\right) \\
& =\theta[0 ; 0]\left(p_{1} \mid 2 i \beta_{1}\right) \theta[0 ; 0]\left(p_{2} \mid 4 i \beta_{2}-2 i \beta_{1}\right)+\theta[1 / 2 ; 1 / 2]\left(p_{1} \mid 2 i \beta_{1}\right) \theta[1 / 2 ; 1 / 2]\left(p_{2} \mid 4 i \beta_{2}-2 i \beta_{1}\right),
\end{aligned}
$$

where $s \in\{-1 ; 0 ; 1\}, \mathcal{U}\left(\mathcal{P}_{\infty}^{+}\right)-\mathbf{Z}_{0} \equiv \mathbf{Z}=\left(z_{1}, z_{2}\right)^{t} \in \mathbb{R}^{2}$,

$$
\begin{aligned}
& p_{1}=z_{1}+2 \sum_{j \geqslant 1} v_{2 j} t_{2 j-1}+s\left(\frac{1}{2}-i \beta_{1}\right), \\
& p_{2}=z_{1}-2 z_{2}-2 i v_{1} x-2 i \sum_{j \geqslant 1} v_{2 j+1} t_{2 j}-s\left(\frac{1}{2}+i \beta_{1}+2 i \delta_{2}\right),
\end{aligned}
$$

admits the following reductions

$$
f_{0}^{*}(\mathbf{x})=f_{0}(\widehat{J} \mathbf{x}), \quad f_{1}^{*}(\mathbf{x})=f_{-1}(\widehat{J} \mathbf{x}), \quad f_{-1}^{*}(\mathbf{x})=f_{1}(\widehat{J} \mathbf{x}) .
$$

Hence, each solution (2.10) of equations in AKNS hierarchy constructed by curve (5.4) satisfies the reduction

$$
q^{*}(\mathbf{x})=-p(\widehat{J} \mathbf{x})
$$

for all $z_{1}, z_{2} \in \mathbb{R}$.

5.3. Two-phase solution. Case 3. The calculations made for the spectral curve

$$
\chi^{2}=\left(\lambda^{2}-a^{2}\right)\left(\lambda^{2}-b^{2}\right)\left(\lambda^{2}-c^{2}\right), \quad a, b, c \in \mathbb{R},
$$

give the following formulae

$$
B=\left(\begin{array}{cc}
2 i \beta_{1} & i \beta_{1} \\
i \beta_{1} & i \beta_{2}
\end{array}\right), \quad \Delta=\left(\begin{array}{c}
-i \beta_{1} \\
i \delta_{2}
\end{array}\right), \quad \mathbf{V}^{2 j-1}=\left(\begin{array}{c}
0 \\
i v_{2 j-1}
\end{array}\right), \quad \mathbf{V}^{2 j}=\left(\begin{array}{c}
2 v_{2 j} \\
v_{2 j}
\end{array}\right),
$$

where $\beta_{j}, \delta_{j}, v_{j} \in \mathbb{R}$. Therefore,

$$
\begin{array}{cc}
\widetilde{B}=\left(\begin{array}{cc}
2 i \beta_{1} & 0 \\
0 & 4 i \beta_{2}-2 i \beta_{1}
\end{array}\right), & \widetilde{A}=\left(\begin{array}{cc}
0 & 0 \\
0 & 0
\end{array}\right), \\
\widetilde{\mathbf{V}}^{2 j-1}=\left(\begin{array}{c}
0 \\
-2 i v_{2 j-1}
\end{array}\right), & \widetilde{\mathbf{V}}^{2 j}=\left(\begin{array}{c}
2 v_{2 j} \\
0
\end{array}\right) .
\end{array}
$$


It is easy to confirm that the corresponding two-dimensional theta function

$$
\begin{aligned}
f_{s}(\mathbf{x}) & =\Theta\left(\mathbf{Z}+\mathbf{V}^{1} x+\sum_{j \geqslant 1} \mathbf{V}^{j+1} t_{j}+s \Delta \mid B\right) \\
& =\theta[0 ; 0]\left(p_{1} \mid 2 i \beta_{1}\right) \theta[0 ; 0]\left(p_{2} \mid 4 i \beta_{2}-2 i \beta_{1}\right)+\theta[1 / 2 ; 0]\left(p_{1} \mid 2 i \beta_{1}\right) \theta[1 / 2 ; 0]\left(p_{2} \mid 4 i \beta_{2}-2 i \beta_{1}\right),
\end{aligned}
$$

where $s \in\{-1 ; 0 ; 1\}, \mathcal{U}\left(\mathcal{P}_{\infty}^{+}\right)-\mathbf{Z}_{0} \equiv \mathbf{Z}=\left(z_{1}, z_{2}\right)^{t} \in \mathbb{R}^{2}$,

$$
\begin{aligned}
& p_{1}=z_{1}+2 \sum_{j \geqslant 1} v_{2 j} t_{2 j-1}-i s \beta_{1}, \\
& p_{2}=z_{1}-2 z_{2}-2 i v_{1} x-2 i \sum_{j \geqslant 1} v_{2 j+1} t_{2 j}-i s\left(\beta_{1}+2 \delta_{2}\right),
\end{aligned}
$$

admits reductions (5.5). Therefore, solution (2.10) of the equations in AKNS hierarchy constructed by curve (5.6) satisfies the reduction

$$
q^{*}(\mathbf{x})=p(\widehat{J} \mathbf{x})
$$

for all $z_{1}, z_{2} \in \mathbb{R}$.

5.4. Two-phase solution. Case 4. The calculations made for spectral curve (5.6) give:

$$
B=\left(\begin{array}{cc}
i \beta_{2} & i \beta_{1} \\
i \beta_{1} & i \beta_{2}
\end{array}\right), \quad \Delta=\left(\begin{array}{c}
1-\delta_{2} \\
\delta_{2}
\end{array}\right), \quad \mathbf{V}^{2 j-1}=\left(\begin{array}{c}
-v_{2 j-1} \\
v_{2 j-1}
\end{array}\right), \quad \mathbf{V}^{2 j}=\left(\begin{array}{c}
i v_{2 j} \\
i v_{2 j}
\end{array}\right),
$$

where $\beta_{j}, \delta_{j}, v_{j} \in \mathbb{R}$. Therefore,

$$
\begin{gathered}
\widetilde{B}=\left(\begin{array}{cc}
2 i\left(\beta_{2}+\beta_{1}\right) & 0 \\
0 & 2 i\left(\beta_{2}-\beta_{1}\right)
\end{array}\right), \quad \widetilde{A}=\left(\begin{array}{ll}
0 & 0 \\
0 & 0
\end{array}\right), \\
\widetilde{\mathbf{V}}^{2 j-1}=\left(\begin{array}{c}
0 \\
-2 v_{2 j-1}
\end{array}\right), \quad \widetilde{\mathbf{V}}^{2 j}=\left(\begin{array}{c}
2 i v_{2 j} \\
0
\end{array}\right) .
\end{gathered}
$$

It is easy to confirm that the corresponding two-dimensional theta function

$$
\begin{aligned}
f_{s}(\mathbf{x})= & \Theta\left(\mathbf{Z}+\mathbf{V}^{1} x+\sum_{j \geqslant 1} \mathbf{V}^{j+1} t_{j}+s \Delta \mid B\right) \\
= & \theta[0 ; 0]\left(p_{1} \mid 2 i\left(\beta_{2}+\beta_{1}\right)\right) \theta[0 ; 0]\left(p_{2} \mid 2 i\left(\beta_{2}-\beta_{1}\right)\right) \\
& +\theta[1 / 2 ; 0]\left(p_{1} \mid 2 i\left(\beta_{2}+\beta_{1}\right)\right) \theta[1 / 2 ; 0]\left(p_{2} \mid 2 i\left(\beta_{2}-\beta_{1}\right)\right),
\end{aligned}
$$

where $s \in\{-1 ; 0 ; 1\}, \mathcal{U}\left(\mathcal{P}_{\infty}^{+}\right)-\mathbf{Z}_{0} \equiv i \mathbf{Z}$ and $\mathbf{Z}=\left(z_{1}, z_{2}\right)^{t} \in \mathbb{R}^{2}$,

$$
\begin{aligned}
& p_{1}=i z_{1}+i z_{2}+2 i \sum_{j \geqslant 1} v_{2 j} t_{2 j-1}+s, \\
& p_{2}=i z_{1}-i z_{2}-2 v_{1} x-2 \sum_{j \geqslant 1} v_{2 j+1} t_{2 j}+s\left(1-2 \delta_{2}\right),
\end{aligned}
$$

admits reductions (5.5). Therefore, solution 2.10 of equations in AKNS hierarchy constructed by curve (5.6) under the second choice of the basis of cycles also satisfies the reduction

$$
q^{*}(\mathbf{x})=p(\widehat{J} \mathbf{x})
$$

for all $z_{1}, z_{2} \in \mathbb{R}$. 


\section{CONCLUDING REMARKS}

To construct solutions for one of nonlocal equations in AKNS hierarchy, we can take an arbitrary solutions of local equations in the same hierarchy obeying the conditions

$$
\begin{aligned}
& p(-x, 0,0, \ldots)=p(x, 0,0, \ldots), \\
& q^{*}(\mathbf{x})=\sigma p(\mathbf{x}), \quad x, t_{k} \in \mathbb{R}, \quad \sigma= \pm 1 .
\end{aligned}
$$

Then, in particular, the functions

$$
p\left(x, t, i T_{2}, T_{3}, i T_{4}, \ldots\right), \quad q\left(x, t, i T_{2}, T_{3}, i T_{4}, \ldots\right), \quad T_{k} \in \mathbb{R}
$$

are solutions of $\mathcal{P} \mathcal{T}$-symmetric nonlinear Schrödinger equation

$$
q^{*}(\mathbf{x})=\sigma p(\widehat{J} \mathbf{x})
$$

We note exactly such conditions are satisfied by rogue waves constructed in works [27], [29]. At the same time, the functions

$$
p\left(x, T_{1}, i T_{2}, t, i T_{4}, \ldots\right), \quad q\left(x, T_{1}, i T_{2}, t, i T_{4}, \ldots\right), \quad T_{k} \in \mathbb{R}
$$

are solutions of $\mathcal{P} \mathcal{T}$-symmetric Lakshmanan-Porsezian-Daniel equation.

\section{BIBLIOGRAPHY}

1. V. Konotop, J. Yang, D. Zezulin. Nonlinear waves in PT-symmetric systems // Rev. Modern Phys. 88:3, 035002, (2016).

2. D. Christodoulides, J. Yang, editors. Parity-time symmetry and its applications. Springer, Singapore (2018).

3. M.J. Ablowitz, Z.H. Musslimani. Integrable nonlocal nonlinear Schrödinger equation // Phys. Rev. Let. 110:6, 064105 (2013).

4. M.J. Ablowitz, Z.H. Musslimani. Integrable discrete PT symmetric model // Phys. Rev. E. 90:3, 032912 (2014).

5. M.J. Ablowitz, Z.H. Musslimani. Inverse scattering transform for the integrable nonlocal nonlinear Schrödinger equation// Nonlinearity. 29:3, 915-946 (2016).

6. M.J. Ablowitz, Z.H. Musslimani. Integrable nonlocal nonlinear equations // Stud. Appl. Math. 139:1, 7-59 (2017).

7. T.P. Horikis, M.J. Ablowitz. Rogue waves in nonlocal media // Phys. Rev. E. 95:4, 042211 (2017).

8. M.J. Ablowitz, B.F. Feng, X.D. Luo, Z.H. Musslimani. Reverse space-time nonlocal SineGordon/Sinh-Gordon equations with nonzero boundary conditions // Stud. Appl. Math. 141:3, 267-307 (2018).

9. V.S. Gerdjikov, G.G. Grahovski, R.I. Ivanov. The N-wave equations with PT symmetry // Theor. Math. Phys. 188:3, 1305-1321 (2016).

10. D.Y. Liu, W.R. Sun. Rational solutions for the nonlocal sixth-order nonlinear Schrödinger equation // Appl. Math. Lett. 84, 63-69 (2018).

11. H.Q. Zhang, M. Gao. Rational soliton solutions in the parity-time-symmetric nonlocal coupled nonlinear Schrödinger equations // Comm. Nonlin. Sci. Numer. Sim. 63, 253-260 (2018).

12. Z.X. Zhou. Darboux transformations and global solutions for a nonlocal derivative nonlinear Schrodinger equation // Comm. Nonl. Sci. Numer. Simul. 62, 480-488 (2018).

13. Y. Cao, B. Malomed, J. He. Two (2+1)-dimensional integrable nonlocal nonlinear Schrodinger equations: Breather, rational and semi-rational solutions // Chaos, Solitons and Fractals. 114, 99-107 (2018).

14. B. Yang, Y. Chen. Reductions of Darboux transformations for the PT-symmetric nonlocal DaveyStewartson equations // Appl. Math. Lett. 82, 43-49 (2018).

15. Z.J. Yang, S.M. Zhang, X.L. Li, Z.G. Pang. Variable sinh-Gaussian solitons in nonlocal nonlinear Schrodinger equation // Appl. Math. Lett. 82, 64-70 (2018). 
16. K. Manikandan, Priya N. Vishnu, M. Senthilvelan, R. Sankaranarayanan. Deformation of dark solitons in a PT-invariant variable coefficients nonlocal nonlinear Schrodinger equation // Chaos. 28:8, 083103 (2018).

17. J. Rao, Y. Zhang, A. Fokas, J. He. Rogue waves of the nonlocal Davey-Stewartson I equation // Nonlinearity. 31:9, 4090-4107 (2018).

18. X.Y. Tang, Z.F. Liang, X.Z. Hao. Nonlinear waves of a nonlocal modified KdV equation in the atmospheric and oceanic dynamical system // Comm. Nonl. Sci. Numer. Simul. 60,62-71 (2018).

19. K. Chen, X. Deng, S. Lou, D.J. Zhang. Solutions of nonlocal equations reduced from the AKNS hierarchy // Stud. Appl. Math. 141:1, 113-141 (2018).

20. W. Liu, X. Li. General soliton solutions to a $(2+1)$-dimensional nonlocal nonlinear Schrodinger equation with zero and nonzero boundary conditions // Nonl. Dynam. 93:2, 721-731 (2018).

21. P. Vinayagam, R. Radha, U. Al Khawaja, L. Ling. New classes of solutions in the coupled PT symmetric nonlocal nonlinear Schrodinger equations with four wave mixing // Comm. Nonl. Sci. Numer. Simul. 59, 387-395 (2018).

22. Y. Cao, J. Rao, D. Mihalache, J. He. Semi-rational solutions for the $(2+1)$-dimensional nonlocal Fokas system // Appl. Math. Lett. 80, 27-34 (2018).

23. C. Qian, J. Rao, D. Mihalache, J. He. Rational and semi-rational solutions of the $y$-nonlocal Davey-Stewartson I equation // Computers Math. Appl. 75:9, 3317-3330 (2018).

24. M. Gurses, A. Pekcan. Nonlocal modified KdV equations and their soliton solutions by Hirota method // Comm. Nonl. Sci. Numer. Simul. 67, 427-448 (2019).

25. Q. Zhang, Y. Zhang, R. Ye. Exact solutions of nonlocal Fokas-Lenells equation // Appl. Math. Lett. 98, 336-343 (2019).

26. V.S. Gerdjikov. On the integrability of Ablowitz-Ladik models with local and nonlocal reductions // J. Phys. Conf. Ser. 1205:1, 012015 (2019).

27. B. Yang, J. Yang. Rogue waves in the nonlocal PT-symmetric nonlinear Schrödinger equation // Lett. Math. Phys. 109, 945-973 (2019).

28. J. Yang. General n-solitons and their dynamics in several nonlocal nonlinear Schrödinger equations // Phys. Lett. A. 383, 328-337 (2019).

29. B. Yang, J. Yang. On general rogue waves in the parity-time-symmetric nonlinear Schrodinger equation // J. Math. Anal. Appl. 487:2, 124023 (2019).

30. Y. Yang, T. Suzuki, X. Cheng. Darboux transformations and exact solutions for the integrable nonlocal Lakshmanan-Porsezian-Daniel equation // Appl. Math. Lett. 99, 105998 (2020).

31. A.O. Smirnov, E.E. Aman. The simplest oscillating solutions of nonlocal nonlinear models // J. Phys. Conf. Ser. 1399:2, 022020 (2019).

32. A.O. Smirnov, E.E. Aman. One-phase elliptic solutions of the nonlocal nonlinear equations from AKNS hierarchy and their spectral curves // J. Phys. Conf. Ser. 1515:3, 032080 (2020).

33. V.B. Matveev, A.O. Smirnov. Multiphase solutions of nonlocal symmetric reductions of equations of the AKNS hierarchy: general analysis and simplest examples // Theor. Math. Phys. 204:3, 1154-1165 (2020).

34. V.B. Matveev, A.O. Smirnov. AKNS hierarchy, MRW solutions, $P_{n}$ breathers, and beyond // J. Math. Phys. 59:9, 091419 (2018).

35. V.B. Matveev, A.O. Smirnov. Two-phase periodic solutions to the AKNS hierarchy equations // J. Math. Sci. 242:5, 722-741 (2019).

36. A.R. Its, V.P. Kotlyarov. On a class of solutions of the nonlinear Schrödinger equation // Dokl. Akad. Nauk Ukrain. SSR. Ser. A. 11, 965-968 (1976). (in Russian)

37. V.P. Kotlyarov. Periodic problem for the nonlinear Schrödinger equation // Preprint: arXiv:1401.4445 (2014).

38. E.D. Belokolos, A.I. Bobenko, V.Z. Enol'skii, A.R. Its, V.B. Matveev. Algebro-geometrical approach to nonlinear evolution equations. Springer, Berlin (1994).

39. M.J. Ablowitz, D.J. Kaup, A.C. Newell, H. Segur. The inverse scattering transform-Fourier analysis for nonlinear problems // Stud. Appl. Math. 53:4, 249-315 (1974). 
40. M. Lakshmanan, K. Porsezian, M. Daniel. Effect of discreteness on the continuum limit of the Heisenberg spin chain // Phys. Lett. A. 133:9, 483-488 (1988).

41. K. Porsezian, M. Daniel, M. Lakshmanan. On the integrability aspects of the one-dimensional classical continuum isotropic Heisenberg spin chain // J. Math. Phys. 33, 1807-1816 (1992).

42. M. Daniel, K. Porsezian, M. Lakshmanan. On the integrable models of the higher order water wave equation // Phys. Lett. A. 174:3, 237-240 (1993).

43. A.O Smirnov. Solution of a nonlinear Schrödinger equation in the form of two-phase freak waves // Theor. Math. Phys. 173:1, 1403-1416 (2012).

44. A.O Smirnov. Periodic two-phase "rogue waves"// Math. Notes. 94:6, 897-907 (2013).

45. A.O Smirnov., S.G. Matveenko, S.K. Semenov, E.G. Semenova. Three-phase freak waves // SIGMA. 11, 032 (2015).

46. B.A. Dubrovin. Theta functions and non-linear equations. Russ. Math. Surv. 36:2, 11-92 (1981).

47. J.D. Fay. Theta-functions on Riemann surfaces. Springer, Berlin (1973).

48. A. Krazer. Lehrbuch der Thetafunktionen. Teubner, Leipzig (1903).

49. H.F. Baker. Abel's theorem and the allied theory including the theory of theta functions. Univ. Press, Cambridge (1897).

50. D. Mumford. Tata lectures on theta. I. Birkhäuser, Boston (1983).

51. D. Mumford. Tata lectures on theta. II. Birkhäuser, Boston (1984).

52. A.O. Smirnov. A matrix analogue of the Appell theorem and reduction of multidimensional Riemann theta-functions // Math. USSR Sb. 61:2, 379-388 (1988).

Aleksandr Olegovich Smirnov,

State University of Aerospace Instrumentation,

Bolshaya Morskaya str. 67A,

190000, Saint-Petersburg, Russia

E-mail: alsmir@guap.ru

Vladimir Borisovich Matveev,

St. Petersburg Department

of V.A. Steklov Mathematical Institute

Fontanka emb. 27,

191023, Saint-Petersburg, Russia

E-mail: vladimir.matveev9@gmail.com 